\title{
Publisher Correction: Dissecting and rebuilding the glioblastoma microenvironment with engineered materials
}

Kayla J. Wolf, Joseph Chen, Jason D. Coombes, Manish K. Aghi and Sanjay KumariD

Nature Reviews Materials (2019) https://doi.org/10.1038/s41578-019-0135-y Published online 16 August 2019

The originally published article contained some wrongly numbered references. In particular, in the caption of Figure 2 references 223,224 and 195 should have been 228, 229 and 199, respectively. In the 'Matrigel' row of Table 3, references 16, 119, 167 should have been 118,191 and 192, respectively. This has been corrected both in the PDF and in the HTML versions of the article.

https://doi.org/10.1038/s41578-020-00242-3 I Published online 4 September 2020

๑) Springer Nature Limited 2020 